\title{
Objectively-measured physical activity and stress levels in workers: a systematic review
}

\author{
Atividade física medida objetivamente e níveis de estresse em trabalhadores: uma \\ revisão sistemática
}

AUTHOR'S
Marcelo Frio Marins ${ }^{1}$ (D)
${\text { Barbara Sutil da Silva }{ }^{1} \text { iD }}$
Natan Feter ${ }^{1}$ (D)
Marcelo Cozzensa da Silva ${ }^{1}$ (D)
1 Universidade Federal de Pelotas, Programa de
Pós-Graduação em Educação Física, Pelotas, Rio
Grande do Sul, Brasil.
CORRE SPONDING
Marcelo Frio Marins
marcelofriomarins@hotmail.com
Rua Luís de Camões, n. 625. Pelotas, Rio
Grande do Sul, Brasil.
CEP: 96055-630.
DOI

$10.12820 /$ rbafs.26e 0232

\section{(cc) BY}

This work is licensed under a Creative Commons Attribution 4.0 International License.

\begin{abstract}
To investigate the relationship between objectively measured physical activity and occupational stress in different work environments. This systematic review, registered in the PROSPERO database (CRD42020214884), followed the PRISMA methodology. The search took place in October/2020 in the following databases: Web of Science, SPORTDiscus, MedLine/PubMed, PsycINFO, EMBASE, OVID MEDLINE, Scielo and CINAHL. Keywords related to eligible participants (adults and workers), interventions (physical activity objectively measured), comparison (control group or baseline), outcome (stress), and study design (observational studies) were combined using Boolean terms. From 1,524 identified records, 12 articles were included, totaling 2,082 workers. $66.7 \%$ of the studies were carried out in Europe and $50.0 \%$ among health professionals. Blue collar workers $(20.7 \%[\mathrm{n}=430])$ and white collar workers $(18.3 \%[\mathrm{n}=382])$, medical resident $(6.5 \%[\mathrm{n}=135])$ and protection services $(9.7 \%[\mathrm{n}=202])$ were the predominant occupations. Physical activity was higher in blue-collar workers than in white-collar workers, and shift-working nurses were more active compared to non-shift workers and office workers. Increased mental workload was not associated with time spent on physical activities in most studies (10 [83.3\%)]). Some studies showed that light physical activity was associated with higher levels of stress and moderate to vigorous physical activity was beneficial for reducing stress dimensions. In conclusion, most studies did not find an association between objectively measured physical activity and the level of stress in workers. Studies with robust methodologies and covering different groups of workers remain necessary.
\end{abstract}

Keywords: Workers; Physical activity; Stress.

\section{RESUMO}

Investigar a relação entre a atividade física medida objetivamente e o estresse ocupacional em diferentes ambientes de trabalho. Esta revisão sistemática, registrada na base de dados PROSPERO (CRD42020214884), seguiu a metodologia PRISMA. A busca ocorreu em outubro/2020 nas bases de dados: Web of Science, SPORTDiscus, MedLine/PubMed, PsycINFO, EMBASE, OVID MEDLINE, Scielo e CINAHL. Palavras-chave relacionadas aos participantes elegiveis (adultos e trabalhadores), intervençôes (atividade física medida objetivamente), comparação (grupo de controle ou medida basal), resultado (estresse) e desenho do estudo (estudos observacionais) foram combinadas usando termos booleanos. De 1.524 registros identificados, 12 artigos foram incluídos, totalizando 2.082 trabalhadores. 66, 7\% dos estudos foram realizados na Europa e 50,0\% entre profissionais de saúde. Trabalhadores "colarinho azul" $(20,7 \%[n=430])$ e "colarinho branco" $(18,3 \%[n=382])$, residente médico $(6,5 \%[n=135])$ e serviços de proteção $(9,7 \%[n=202])$ foram as ocupações predominantes. A atividade física foi maior em trabalhadores "colarinho azul" do que em "colarinho branco", e as enfermeiras que trabalhavam por turnos foram mais ativas comparadas as que não trabalhavam em turnos e a funcionários de escritório. O aumento da carga mental não foi associado ao tempo gasto em atividades físicas na maioria dos estudos (10 [83,3\%]). Alguns estudos mostraram que a atividade física leve foi associada a maiores niveis de estresse e a moderada a vigorosa benéfica para redução das dimensões de estresse. Em conclusão, a maioria dos estudos não encontrou associação entre atividade física medida objetivamente com o nivel de estresse em trabalhadores. Estudos com metodologias robustas e abrangendo diferentes grupos de trabalhadores permanecem necessários.

Palavras-chave: Trabalhadores; Atividade física; Estresse

\section{Introduction}

Physical inactivity is an important risk factor for morbidity and premature mortality. In fact, around 5.2 million people deaths worldwide were attributable to physical inactivity in $2008^{1}$. Achieving the recommendation of 150 minutes of moderate-to-vigorous physical activity per week ${ }^{2}$ is related to reduced risk for a myriad number of chronic conditions such as ischemic heart 
disease, diabetes, stroke, breast cancer, and colon can$\mathrm{cer}^{3}$. On the other hand, this relationship seems to shift when accounting for physical activity performed during working time. For example, workers with high physical demands during occupational time have a higher risk of ischemic heart disease ${ }^{4}$, atrial fibrillation ${ }^{5}$, and premature mortality ${ }^{6}$. The contrasting effect of occupational physical activity is called the physical activity paradox ${ }^{7}$.

Besides the elevated physical demand, some workers might be exposed to occupational stress, leading to mental disorders such as anxiety, depression, and Burnout syndrome ${ }^{8,9}$. Some mechanisms such as elevated heart rate over long periods ${ }^{10}$, environmental and psycho-social stressors ${ }^{11}$, and low-to-high intensity occupational physical activity without sufficient recovery ${ }^{12}$ are associated with detrimental effects in both the mental and physical health of this population. All these factors seem to induce chronic inflammation intimately associated with disorders such as obesity, diabetes, arthritis, and sleep impairment ${ }^{13}$.

Although the literature has extensively documented the beneficial effect of general physical activity in psychological stress at population level ${ }^{14}$, few studies address the relationship between physical activity and occupational stress. Considering the "physical activity paradox" previously mentioned, a broader understanding of the role of different domains of physical activity in occupational stress is warranted. However, no systematic reviews on this subject were found. Therefore, the aim of this study was to investigate the association between objectively-measured physical activity and occupational stress in different work environments.

\section{Methods}

\section{Protocol and registration}

The protocol was prospectively registered on the PROSPERO International Prospective Register for Systematic Reviews website (CRD42020214884). Preferred Reporting Items for Systematic Reviews and Meta-Analyzes (PRISMA) was used to ensure that methodological aspects and results were conducted and reported systematically ${ }^{15}$.

\section{Eligibility criteria}

PICOS criteria were determined before the database search based on population, intervention, control, outcome, and study design.

- Population: Workers aged 18 or older with no re- strictions for health conditions.

- Indicator: Physical activity objectively measured by accelerometer or pedometer.

- Control: No control group required.

- Outcome: Psychological stress.

- Study: Observational studies were included.

\section{Information sources and search}

A systematic review of the literature was conducted to find studies that aimed to improve stair use among adult population. The search was conducted in October 2020 and included the following databases: Web of Science, SPORTDiscus, MedLine/PubMed, PsycINFO, EMBASE, OVID MEDLINE, Scielo and CINAHL. Keywords related to eligible participants (adults and workers), interventions (objective-measured physical activity), comparison (control group or baseline measure), outcome (stress), and study design (observational studies) were combined as shown in Appendix 1. Keywords were used in the English language. There was no time limitation for publication studies. Detailed search strategy can be found in the Appendix 1 .

\section{Study selection}

All articles were included and exported to Mendeley Desktop reference management software (1.19.4 version). Abstract and titles identified in electronic databases were checked by two independent reviewers (MFM and BSS) to include eligible papers in full-text analyzes. A third independent reviewer (NF) was called in case of disagreement to solve any issue.

\section{Data collection process}

Data extraction was conducted independently by two reviewers (MFM and BBS). Country, sample size and characteristics, physical activity (method, domain, and findings), stress (method and findings), and main conclusions were extracted to a standardized form. Physical activity as a continuous variable (minutes per week, steps per day, counts per minute) as well as lower and upper confidence interval limits were obtained as effect size.

\section{Risk of bias}

The quality of selected studies was assessed by two independent reviewers (MFM and BBS) using a quality rating list based on a previously published checklist for reporting of observational studies ${ }^{16,17}$. The detailed quality ranking list contains 15 items that assess diffe- 
rent methodological aspects, as previously published ${ }^{18}$ and ilustrated in Table 1.

Table 1 - Criteria for quality assessment of studies

\begin{tabular}{|c|c|c|}
\hline Item & Criterion & Description \\
\hline 1 & Objectives & $\begin{array}{l}\text { Are the objectives or hypotheses of the } \\
\text { research described in the paper stated? }\end{array}$ \\
\hline 2 & Study design & Is the study design presented? \\
\hline $3 a$ & Target population & $\begin{array}{l}\text { Do the authors describe the target } \\
\text { population they wanted to research? }\end{array}$ \\
\hline $3 b$ & Sample & $\begin{array}{l}\text { Was a random sample of the target } \\
\text { population taken? AND was the response } \\
\text { rate } 60 \% \text { or more? }\end{array}$ \\
\hline $3 c$ & Sample & Is participant selection described? \\
\hline $3 \mathrm{~d}$ & Sample & $\begin{array}{l}\text { Is participant recruitment described, or } \\
\text { referred to? }\end{array}$ \\
\hline $3 e$ & Sample & $\begin{array}{l}\text { Are the inclusion and/or exclusion criteria } \\
\text { stated? }\end{array}$ \\
\hline $3 f$ & Sample & $\begin{array}{l}\text { Is the study sample described? (minimum } \\
\text { descriptionsample size, gender, age and an } \\
\text { indicator of SES) }\end{array}$ \\
\hline $3 g$ & Sample & $\begin{array}{l}\text { Are the numbers of participants at each } \\
\text { stage of the study reported? (Authors should } \\
\text { report at least numbers eligible, numbers } \\
\text { recruited, numbers with data at baseline, and } \\
\text { numbers lost to follow-up) }\end{array}$ \\
\hline 4 & Variables & $\begin{array}{l}\text { Are the measures of occupational sitting and } \\
\text { the health outcome described? }\end{array}$ \\
\hline $5 \mathrm{a}$ & $\begin{array}{l}\text { Data sources and } \\
\text { collection }\end{array}$ & $\begin{array}{l}\text { Do authors describe the source of their data } \\
\text { (e.g., cancer registry, health survey) AND } \\
\text { did authors describe how the data were } \\
\text { collected? (e.g., by mail) }\end{array}$ \\
\hline $5 b$ & Measurement & $\begin{array}{l}\text { Was reliability of the measure(s) of } \\
\text { occupational sitting mentioned or referred } \\
\text { to? }\end{array}$ \\
\hline $5 c$ & Measurement & $\begin{array}{l}\text { Was the validity of the measure(s) of } \\
\text { occupational sitting mentioned or referred } \\
\text { to? }\end{array}$ \\
\hline $6 a$ & $\begin{array}{l}\text { Statistical } \\
\text { methods }\end{array}$ & $\begin{array}{l}\text { Were appropriate statistical methods used } \\
\text { and described, including those for addressing } \\
\text { confounders? }\end{array}$ \\
\hline $6 \mathrm{~b}$ & $\begin{array}{l}\text { Statistical } \\
\text { methods }\end{array}$ & $\begin{array}{l}\text { Were the numbers/percentages of } \\
\text { participants with missing data for sitting and } \\
\text { the health outcome indicated AND If more } \\
\text { than } 20 \% \text { of data in the primary analyses } \\
\text { were missing, were methods used to address } \\
\text { missing data? }\end{array}$ \\
\hline
\end{tabular}

\section{Results}

A pilot analysis was conducted to check the agreement between the scores from both researchers. A Cohen's kappa statistic equal to 1.00 and $100.0 \%$ of agreement was achieved between raters. The items are rated as yes ( 1 point), no (0 points), or unclear (0 points). As all criteria had the same weight, quality score ranges from zero to 15 for each study.

From 1,524 records identified in six electronic da- tabases, 12 articles were included in this systematic review. The main reasons for exclusion were studies with no workers (50.0\%), no objectively measured physical activity (22.8\%), and no stress assessments (22.5\%), as shown in Figure 1.

Characteristics of the included articles are shown in Table 2. From the 12 included studies, $66.7 \%$ ( $n=$ 8) were carried out in Europe ${ }^{20-26,30}, 16.7 \%(\mathrm{n}=2)$ in America ${ }^{19,29}$, and $16.7 \%(\mathrm{n}=2)$ in $\mathrm{Asia}^{27,28}$. Included articles summed 2,082 workers, which most were health professionals $(50.0 \%[\mathrm{n}=6])^{19,21,25-27,29}$ and women $(56.0 \%[\mathrm{n}=1,166])^{19,21,23,25-28}$. The average age ranged from $22,40 \pm 1,80^{22}$ to $68.15 \pm 9.15^{21}$ yearsold. Blue-collar $(20.7 \%[\mathrm{n}=430])^{24,30}$ and white-collar workers $(18.3 \%[\mathrm{n}=382])^{20,24,28,30}$, medical resident $(6.5 \%[\mathrm{n}=135])^{19,27}$, and protective services $(9.7 \%[\mathrm{n}=$ 202] $)^{22,23}$ were the most frequent occupations.

Regarding the quality of the included studies, the mean score was $11.5 \pm 2.9$, with studies ranging from $5^{19}$ to $14^{20-24}$, shown in Appendix 2 .

Physical activity was assessed by accelerometer and pedometer in $10(83.3 \%)^{20-28}$ and $2(16.7 \%)^{19,29}$ studies respectively. Most studies reported physical activity as minutes per day $(42.9 \%[\mathrm{n}=6])^{19,20,23-25,27}$ or METs per minute $(28.6 \%[n=4])^{26,28-30}$. The mean time spent in

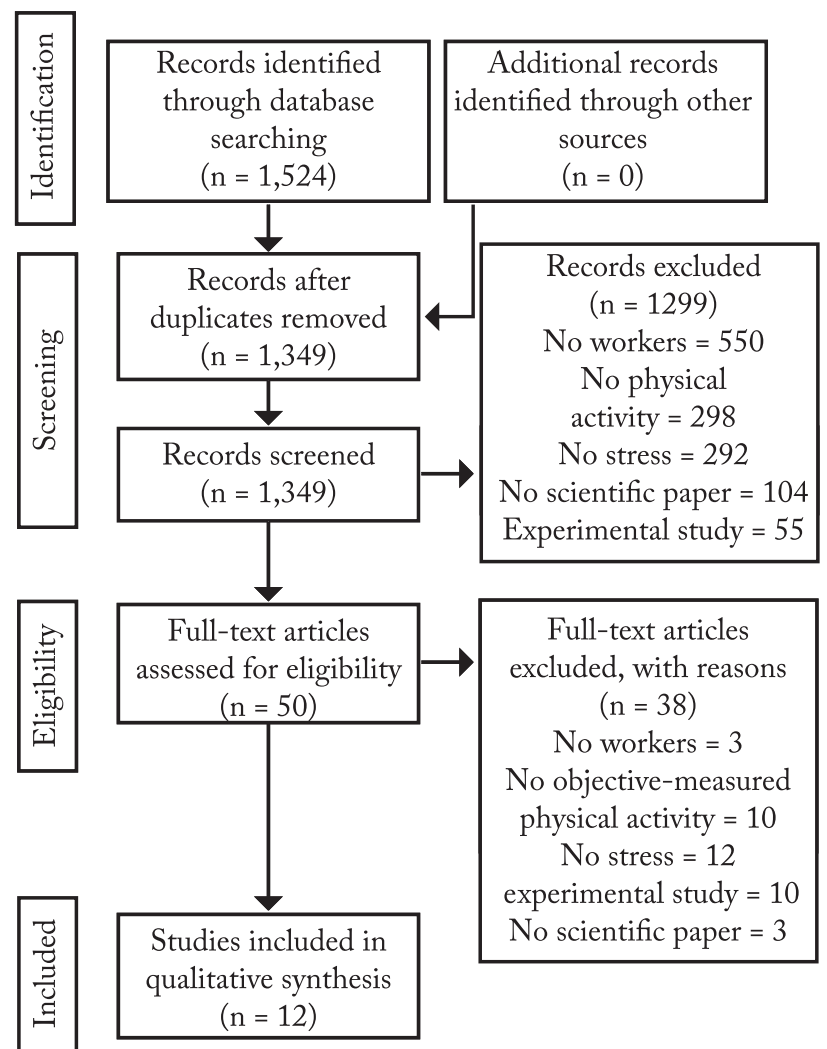

Figure 1-Flow diagram of the studies selected for this systematic review. 
Table 2-Characteristics of included studies $(\mathrm{N}=12)$.

\begin{tabular}{|c|c|c|c|c|c|c|c|c|c|c|c|}
\hline \multirow[b]{2}{*}{ Author } & \multirow[b]{2}{*}{ Country } & \multirow[b]{2}{*}{$\begin{array}{c}\text { Sample/ } \\
\% \\
\text { women }\end{array}$} & \multirow[b]{2}{*}{$\begin{array}{l}\text { Age } \\
\text { (years) }\end{array}$} & \multirow[b]{2}{*}{$\begin{array}{l}\text { Type of } \\
\text { worker }\end{array}$} & \multicolumn{3}{|l|}{ Physical activity } & \multicolumn{4}{|l|}{ Stress } \\
\hline & & & & & Instrument & $\begin{array}{l}\text { Domains } \\
\text { assessed/ } \\
\text { unit }\end{array}$ & $\begin{array}{l}\text { Physical activity } \\
\text { findings }\end{array}$ & Instrument & $\begin{array}{l}\text { Source } \\
\text { assessed }\end{array}$ & Stress findings & Conclusion \\
\hline $\begin{array}{c}\text { Myrtek et } \\
\text { al. }{ }^{30}\end{array}$ & Germany & 86 & $\begin{array}{c}\text { Mean: } \\
50,81\end{array}$ & $\begin{array}{l}\text { White (N } \\
=57) \text { and } \\
\text { blue-collar } \\
(\mathrm{N}=29)\end{array}$ & $\begin{array}{l}\text { Accelerometry/ } \\
23 \text { hours }\end{array}$ & $\begin{array}{l}\text { Work } \\
\text { and } \\
\text { leisure } \\
\text { time/ } \\
\text { units per } \\
\text { min }\end{array}$ & $\begin{array}{l}\text { Blue-collar } \\
\text { workers were } \\
\text { more active } \\
\text { during work; } \\
\text { activities during } \\
\text { leisure-time were } \\
\text { similar between } \\
\text { groups. }\end{array}$ & $\begin{array}{l}\text { Single } \\
\text { question }\end{array}$ & Total & $\begin{array}{l}\text { "White-collar" } \\
\text { workers reported } \\
\text { higher stress levels } \\
\text { at and outside } \\
\text { work than "blue- } \\
\text { collar" workers. }\end{array}$ & $\begin{array}{l}\text { Physical } \\
\text { activity } \\
\text { was not } \\
\text { associated } \\
\text { with stress } \\
\text { levels. }\end{array}$ \\
\hline $\begin{array}{c}\text { Martinez et } \\
\text { al. }^{25}\end{array}$ & $\begin{array}{c}\text { Switzer- } \\
\text { land }\end{array}$ & $\begin{array}{c}18 \\
/ 61.1 \%\end{array}$ & $\begin{array}{l}\text { Mean: } \\
\text { 33,00; } \\
\text { Range: } \\
\text { 29-50 }\end{array}$ & $\begin{array}{l}\text { Obstetricians } \\
\text { at hospitals } \\
\text { of the } \\
\text { Geneva } \\
\text { University }\end{array}$ & $\begin{array}{l}\text { Accelerometry/ } \\
9 \text { hours/day }\end{array}$ & $\begin{array}{l}\text { Total / } \\
\text { steps per } \\
\text { day }\end{array}$ & $\begin{array}{l}\text { Average (range): } \\
7132 \text { (5283-8649) } \\
\text { steps per day; } \\
\text { median (range): } \\
32 \text { min (19-49 } \\
\text { min) moderate- } \\
\text { to-vigorous } \\
\text { physical activity } \\
\text { per day. }\end{array}$ & $\begin{array}{l}\text { MSP-9; visual } \\
\text { analogic scale }\end{array}$ & Work & $\begin{array}{l}\text { No differences } \\
\text { in stress level } \\
\text { between junior and } \\
\text { seniors' workers } \\
\text { were observed. } \\
\text { However, stress } \\
\text { scores were higher } \\
\text { after clinical } \\
\text { appointments in } \\
\text { both groups. }\end{array}$ & $\begin{array}{l}\text { Physical } \\
\text { activity } \\
\text { was not } \\
\text { associated } \\
\text { with stress } \\
\text { levels. }\end{array}$ \\
\hline $\begin{array}{c}\text { Roskoden et } \\
\text { al. }^{26}\end{array}$ & Germany & $\begin{array}{l}\mathrm{N}=44 \\
/ 77.3 \%\end{array}$ & $\begin{array}{c}\text { Shifting= } \\
31,00 \\
\pm 1,90 ; \\
\text { Non- } \\
\text { shifting } \\
=41,62 \pm \\
2,00\end{array}$ & $\begin{array}{l}\text { Nursers } \\
\text { and office } \\
\text { workers }\end{array}$ & $\begin{array}{l}\text { Accelerometry/ } \\
7 \text { consecutives } \\
\text { days }\end{array}$ & $\begin{array}{l}\text { Total / } \\
\text { METs } \\
\text { per min }\end{array}$ & $\begin{array}{l}\text { Shifting workers } \\
\text { were more active } \\
\text { than non-shifting } \\
\text { workers }(2.1 \text { vs. } \\
1.7 \text { METs, p < } \\
0.01)\end{array}$ & $\begin{array}{l}\text { Trier } \\
\text { Inventory } \\
\text { for Chronic } \\
\text { Stress (TICS) }\end{array}$ & Total & $\begin{array}{l}\text { Social burden in } \\
\text { shifts nurses was } \\
\text { greatly increased } \\
\text { compared to the } \\
\text { subgroup of office } \\
\text { workers who do } \\
\text { not work in shifts. } \\
\text { The differences } \\
\text { were greater when } \\
\text { comparing nurses } \\
\text { who work in shifts } \\
\text { with office workers. }\end{array}$ & $\begin{array}{l}\text { Physical } \\
\text { activity } \\
\text { was not } \\
\text { associated } \\
\text { with stress } \\
\text { levels. }\end{array}$ \\
\hline Low et al. ${ }^{27}$ & $\begin{array}{l}\text { Singa- } \\
\text { pore }\end{array}$ & $\begin{array}{c}\mathrm{N}=49 / \\
57.1 \%\end{array}$ & $\begin{array}{l}\text { Mean: } \\
25,00\end{array}$ & Residents & $\begin{array}{l}\text { Accelerometry/ } \\
4 \text { months }\end{array}$ & $\begin{array}{l}\text { Total / } \\
\text { steps per } \\
\text { min per } \\
\text { day }\end{array}$ & $\begin{array}{l}\text { Steps per day } \\
\text { and duration of } \\
\text { physical activity } \\
\text { was similar in } \\
\text { both groups }\end{array}$ & $\begin{array}{l}\text { ProQOL } \\
\text { scale }\end{array}$ & Work & $\begin{array}{l}\text { No difference in } \\
\text { stress level was } \\
\text { observed between } \\
\text { groups. }\end{array}$ & $\begin{array}{l}\text { Physical } \\
\text { activity } \\
\text { was not } \\
\text { associated } \\
\text { with stress } \\
\text { levels. }\end{array}$ \\
\hline $\begin{array}{c}\text { Driesman et } \\
\text { al. }^{19}\end{array}$ & $\begin{array}{l}\text { United } \\
\text { States }\end{array}$ & $\begin{array}{c}\mathrm{N}=27 / \\
25.9 \%\end{array}$ & $\begin{array}{l}\text { Mean } \\
28,90\end{array}$ & $\begin{array}{l}\text { Orthopedics } \\
\text { Residents }\end{array}$ & $\begin{array}{l}\text { Fitbit Flex } \\
\text { (wrist)/ } 4 \\
\text { weeks }\end{array}$ & $\begin{array}{l}\text { Total / } \\
\text { steps per } \\
\text { day }\end{array}$ & $\begin{array}{l}\text { Orthopedics } \\
\text { residents were } \\
\text { more active than } \\
\text { other residents }\end{array}$ & $\begin{array}{l}\text { MBI e } \\
\text { PHQ-9 }\end{array}$ & Total & $\begin{array}{l}\text { Orthopedic } \\
\text { residents reported } \\
\text { significantly higher } \\
\text { rates of emotional } \\
\text { exhaustion and } \\
\text { depersonalization } \\
\text { than other } \\
\text { residents. }\end{array}$ & $\begin{array}{l}\text { Physical } \\
\text { activity } \\
\text { was not } \\
\text { associated } \\
\text { with stress } \\
\text { levels. }\end{array}$ \\
\hline $\begin{array}{l}\text { Mendelsohn } \\
\text { et al. }{ }^{29}\end{array}$ & Canada & $\mathrm{N}=59$ & $\begin{array}{l}\text { Mean: } \\
28,53 \pm \\
3,00\end{array}$ & $\begin{array}{l}\text { Surgeons, } \\
\text { Clinical } \\
\text { Physicians, } \\
\text { and } \\
\text { Anesthetists } \\
\text { and } \\
\text { Radiologists }\end{array}$ & $\begin{array}{l}\text { FITBit Charge } \\
\text { HR / } 14 \text { days }\end{array}$ & $\begin{array}{l}\text { Work / } \\
\text { METs }\end{array}$ & $\begin{array}{l}\text { Surgical residents } \\
\text { worked the most } \\
\text { hours per week, } \\
\text { followed by } \\
\text { medical and RCD } \\
\text { residents. }\end{array}$ & $\begin{array}{l}\text { Short-Form } \\
\text { Health } \\
\text { Survey e } \\
\text { Maslach } \\
\text { Burnout } \\
\text { Inventory }\end{array}$ & Total & $\begin{array}{l}\text { Scores from } \\
\text { Maslach Burnout } \\
\text { Inventory were } \\
\text { not significantly } \\
\text { different among } \\
\text { groups. }\end{array}$ & $\begin{array}{l}\text { Physical } \\
\text { activity } \\
\text { was not } \\
\text { associated } \\
\text { with stress } \\
\text { levels. }\end{array}$ \\
\hline $\begin{array}{c}\text { Kitano et } \\
\text { al. }^{28}\end{array}$ & Japan & $\begin{array}{c}\mathrm{N}= \\
1095 \\
/ 68.6 \\
\text { women }\end{array}$ & $\begin{array}{c}\text { Mean: } \\
50,20 \pm \\
9,50\end{array}$ & $\begin{array}{l}\text { "White- } \\
\text { collar" } \\
\text { workers }\end{array}$ & $\begin{array}{l}\text { Accelerometry } \\
\text { / at least } 10 \\
\text { days including } \\
\text { work and } \\
\text { weekend days. }\end{array}$ & $\begin{array}{l}\text { Total / } \\
\text { METs }\end{array}$ & $\begin{array}{l}\text { SB and LPA } \\
\text { were positively } \\
\text { associated with } \\
\text { psychological } \\
\text { distress and low } \\
\text { engagement at } \\
\text { work. MVPA was } \\
\text { not associated } \\
\text { with mental } \\
\text { health. }\end{array}$ & $\begin{array}{l}\text { Kessler-10 } \\
\text { (scale of } \\
\text { psychological } \\
\text { suffering) }\end{array}$ & Total & $\begin{array}{l}\text { Levels of } \\
\text { psychological } \\
\text { suffering: } 72.5 \% \text { at } \\
\text { low, } 27.5 \% \text { at high }\end{array}$ & $\begin{array}{l}\text { Physical } \\
\text { activity was } \\
\text { associated } \\
\text { with stress } \\
\text { levels. }\end{array}$ \\
\hline
\end{tabular}


Continue of Table 2 - Characteristics of included studies $(\mathrm{N}=12)$.

\begin{tabular}{|c|c|c|c|c|c|c|c|c|c|c|c|}
\hline \multirow[b]{2}{*}{ Author } & \multirow[b]{2}{*}{ Country } & \multirow[b]{2}{*}{$\begin{array}{c}\text { Sample/ } \\
\% \\
\text { women }\end{array}$} & \multirow[b]{2}{*}{$\begin{array}{l}\text { Age } \\
\text { (years) }\end{array}$} & \multirow[b]{2}{*}{$\begin{array}{l}\text { Type of } \\
\text { worker }\end{array}$} & \multicolumn{3}{|l|}{ Physical activity } & \multicolumn{4}{|l|}{ Stress } \\
\hline & & & & & Instrument & $\begin{array}{l}\text { Domains } \\
\text { assessed/ } \\
\text { unit }\end{array}$ & $\begin{array}{l}\text { Physical activity } \\
\text { findings }\end{array}$ & Instrument & $\begin{array}{l}\text { Source } \\
\text { assessed }\end{array}$ & Stress findings & Conclusion \\
\hline $\begin{array}{c}\text { Marquez et } \\
\text { al. }{ }^{21}\end{array}$ & Belgium & $\begin{array}{c}\mathrm{N}=73 / \\
69.4 \\
\text { women }\end{array}$ & $\begin{array}{l}\text { Mean: } \\
68,15 \pm \\
9,15\end{array}$ & $\begin{array}{l}\text { Caregivers } \\
(\mathrm{N}=24) ; \\
\text { non- } \\
\text { caregivers } \\
(\mathrm{N}=49)\end{array}$ & $\begin{array}{l}\text { Accelerometry } \\
/ 7 \text { consecutive } \\
\text { days. }\end{array}$ & & $\begin{array}{l}\text { No difference } \\
\text { was observed } \\
\text { in time spent } \\
\text { with physical } \\
\text { activity between } \\
\text { caregivers and } \\
\text { non-caregivers. }\end{array}$ & $\begin{array}{l}\text { Perceived } \\
\text { Stress Scale }\end{array}$ & Total & $\begin{array}{l}\text { Caregivers } \\
\text { reported } \\
\text { greater anxiety, } \\
\text { depression, } \\
\text { stress, and } \\
\text { negative } \\
\text { health } \\
\text { symptoms } \\
\text { compared } \\
\text { to non- } \\
\text { caregivers. }\end{array}$ & $\begin{array}{l}\text { Physical } \\
\text { activity was } \\
\text { associated } \\
\text { with stress } \\
\text { levels. }\end{array}$ \\
\hline $\begin{array}{l}\text { Larsson et } \\
\text { al. }^{20}\end{array}$ & Sweden & $\mathrm{N}=314$ & $\begin{array}{c}\text { Mean: } \\
42,20 \pm \\
21,00\end{array}$ & $\begin{array}{l}\text { "White- } \\
\text { collar" } \\
\text { workers }\end{array}$ & $\begin{array}{l}\text { Accelerometry/ } \\
600 \text { min per } \\
\text { day for at least } \\
4 \text { days }\end{array}$ & $\begin{array}{l}\text { Total / } \\
\text { Counts } \\
\text { per min }\end{array}$ & $\begin{array}{l}\text { High-demand } \\
\text { activities were } \\
\text { associated with } \\
\text { vigorous-intensity } \\
\text { physical activity }\end{array}$ & $\begin{array}{l}\text { Likert- } \\
\text { scale in one } \\
\text { question }\end{array}$ & Work & $\begin{array}{l}\text { Low } \\
\text { psychological } \\
\text { stress was } \\
\text { associated } \\
\text { with low } \\
\text { time spent } \\
\text { with vigorous } \\
\text { physical } \\
\text { activity }\end{array}$ & $\begin{array}{l}\text { Physical } \\
\text { activity was } \\
\text { associated } \\
\text { with stress } \\
\text { levels. }\end{array}$ \\
\hline $\begin{array}{l}\text { Schilling et } \\
\text { al. }^{23}\end{array}$ & $\begin{array}{l}\text { Switzer- } \\
\text { land }\end{array}$ & $\begin{array}{c}\mathrm{N}= \\
401 / \\
57.4 \%\end{array}$ & $\begin{array}{l}\text { Range: } \\
20-64\end{array}$ & $\begin{array}{l}\text { Police } \\
\text { officers }\end{array}$ & $\begin{array}{l}\text { Accelerometry } \\
\text { / } 5 \text { days with } \\
10 \text { valid hours }\end{array}$ & $\begin{array}{l}\text { Total / } \\
\text { min per } \\
\text { week }\end{array}$ & $\begin{array}{l}\text { Physical activity } \\
\text { was } 3-7 \text { times } \\
\text { greater than the } \\
\text { WHO guidelines }\end{array}$ & $\begin{array}{l}\text { Job Content } \\
\text { Questionnaire; } \\
\text { Effort-Reward } \\
\text { Imbalance } \\
\text { questionnaire }\end{array}$ & Total & $\begin{array}{l}51.5 \% \text { of } \\
\text { participants } \\
\text { reported } \\
\text { work stress } \\
\text { with possible } \\
\text { adverse } \\
\text { effects }\end{array}$ & $\begin{array}{l}\text { Physical } \\
\text { activity } \\
\text { was not } \\
\text { associated } \\
\text { with stress } \\
\text { levels. }\end{array}$ \\
\hline $\begin{array}{l}\text { Meina et } \\
\text { al. }^{22}\end{array}$ & Poland & $N=26$ & $\begin{array}{l}\text { Mean: } \\
22,40 \\
\pm 1,80\end{array}$ & Firefighters & $\begin{array}{l}\text { Accelerometry } \\
\text { / 24-hour cycle }\end{array}$ & Clusters & - & $\begin{array}{l}\text { Self-reported } \\
\text { questionnaire }\end{array}$ & Work & $\begin{array}{l}\text { Most } \\
\text { participants } \\
\text { reported } \\
\text { none or low } \\
\text { level of stress } \\
\text { perception }\end{array}$ & $\begin{array}{l}\text { Physical } \\
\text { activity } \\
\text { was not } \\
\text { associated } \\
\text { with stress } \\
\text { levels. }\end{array}$ \\
\hline $\begin{array}{l}\text { De Cocker } \\
\text { et al. }{ }^{24}\end{array}$ & Belgium & $\mathrm{N}=401$ & $\begin{array}{l}\text { Range: } \\
20-64\end{array}$ & $\begin{array}{l}\text { White- } \\
\text { collar: } 57.3 \% \\
\text { Blue-collar: } \\
42.7 \%\end{array}$ & $\begin{array}{l}\text { Accelerometry } \\
/ 2-4 \\
\text { consecutive } \\
\text { days }\end{array}$ & $\begin{array}{l}\text { Work, } \\
\text { leisure, } \\
\text { and total } \\
\text { /Hours } \\
\text { per day }\end{array}$ & $\begin{array}{l}\text { Mean of } 1.1 \text { and } \\
0.7 \text { hours/day in } \\
\text { MVPA at work } \\
\text { and leisure-time, } \\
\text { respectively. } \\
\text { Mean time spent } \\
\text { in sedentary } \\
\text { activities was } 7.2 \\
\text { hours/day }\end{array}$ & $\begin{array}{l}\text { General } \\
\text { Health } \\
\text { Questionnaire } \\
(\text { GHQ-12) }\end{array}$ & Total & $\begin{array}{l}\text { Around 35\% } \\
\text { reported } \\
\text { emotional } \\
\text { distress }\end{array}$ & $\begin{array}{l}\text { Physical } \\
\text { activity and } \\
\text { sedentary } \\
\text { behavior } \\
\text { was not } \\
\text { associated } \\
\text { with stress } \\
\text { levels. }\end{array}$ \\
\hline
\end{tabular}

each intensity of physical activity, in minutes per day, by occupational groups is shown in Figure 2. Physical activity was higher in "blue-collar" workers than in "white-collar" ${ }^{30}$, and shift-work nurses were more active than non-shift counterparts and office workers ${ }^{26}$. Likewise, On the other hand, no difference in time spent with physical activity was observed between caregivers and non-caregivers ${ }^{21}$. Among health workers, general and orthopedic surgery residents ${ }^{19,29}$ were more active than internal medicine and neurology, anesthesia and radiology ${ }^{30}$, and other residents ${ }^{19}$.

Different instruments were used among all includ- ed studies to assess stress levels, so quantitative analysis on this outcome was not possible. Workers who experienced higher levels of stress among their peers were white-collar compared to blue-collar workers ${ }^{30}$, shift-working nurses had a higher social burden than office workers ${ }^{26}$, orthopedic residents had higher rates of emotional exhaustion and depersonalization than did residents from other areas ${ }^{19}$ and caregivers reported higher stress values than non-caregivers ${ }^{21}$. The heightened mental burden was not associated with time spent in physical activities in most (10 [83.3\%]) of the included studies ${ }^{19-25,27,29,30}$. In these studies, physical activity 


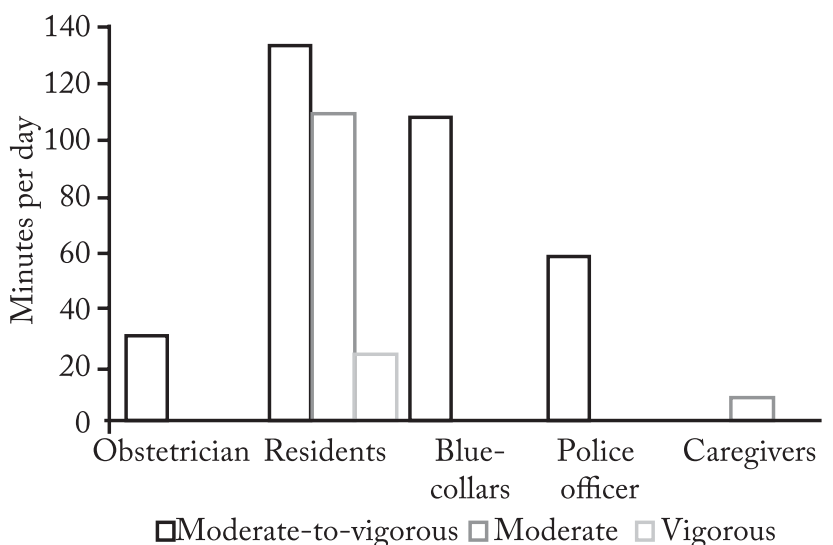

Figure 2 - Minutes per day spent in physical activity stratified by intensity and occupational groups.

was assessed by accelerometer and pedometer among security (fireman and police officers), health (i.e., physicians and image technicians), and general workers.

In studies in which physical activity was associated with heightened stress levels, samples were composed of office workers and nurses. Roskoden et al. ${ }^{26}$ reported that shifting nursing exhibited higher physical activity levels and social overload than non-shifting office among. Similarly, Kitano et al. ${ }^{29}$ revealed that light-intensity physical activity performed on workdays was associated with an increased risk of psychological distress among workers from a life insurance company in Japan. In a final sample of 2,081 workers, we observed that in most studies, physical activity was not associated with stress levels in different occupational groups.

\section{Discussion}

Occupational physical activity has been recently associated with adverse health outcomes including premature mortality ${ }^{6}$. This so-called physical activity paradox was based on the increased physical and mental burden in certain occupational groups such as blue-collar workers $^{11}$. Although "blue-collar" workers had higher time spent with physical activity during work, this difference does not seem to lead to increased stress levels ${ }^{30}$. Nevertheless, these workers spent almost half of the waking time in sedentary activities without physical activity that could attenuate the heightened risk of all-cause mortality associated with such behavior ${ }^{31}$. The absence of the association between occupational physical activity and stress must not offset the importance of promoting physical activity for this group. Understanding the "24-hour activity cycle ${ }^{32}$, which consists of sleep, sedentary behavior, light-intensity physical activity and moderate-to-vigorous physical activity, is critical to promoting a more active lifestyle in workers.

Furthermore, the effect of occupational physical activity on stress levels might not be exclusively related to the type of worker such as "blue-collar" or "white-collar". Shift-workers (i.e., nurses) showed higher METs while working and higher psychological stress than non-shift-workers. Also, physical activity performed by workers in a life insurance company was associated with an increased risk of psychological distress. Likewise, Tejada et al. ${ }^{25}$ reported that the elevated stress levels observed among obstetricians were followed by increased concentration of adrenalin and reduced heart rate variability after a labor suite shift. This modification in the physiological profile indicates an elevated burden of occupational activity in this group of workers. However, the number of steps per day did not differ among off work days, outpatient clinic, and labor suite.

Also, although caregivers reported higher mental distress such as anxiety and stress than non-caregivers, no difference was observed in physical activity level between groups ${ }^{21}$. Altogether, the psychological demand of some types of occupational activities such as the persisted alarming state in the hospital environment and goal-based jobs such as in life insurance companies might trigger emotional distress in workers, with occupational physical activity, even at low intensities exacerbating such adverse effects.

Regarding the quality, all included articles clearly reported objectives, target population (i.e., workers), and the number of participants. However, no study had randomly selected the sample from the target population. Also, only six $(50.0 \%)$ studies $^{20-24,28}$ provided details of the included participants whilst eight $(66.7 \%)^{20-}$ 25,28,30 reported using appropriated statistical methods. The occupational environment has been the focus of the physical activity field for centuries ${ }^{33}$. On the other hand, the remaining gaps in the association between physical activity during work and physical and mental health must be filled by well-design studies with a robust methodology. Asking workers to take part in our studies yields the responsibility to return with important and trustful results. Otherwise, it is just another task to be completed during the occupational time.

Some limitations need to be acknowledged. First, stress was assessed as a self-reported perception. However, biological markers such as cortisol concentration might provide a non-specific viewpoint of the psychological burden of occupational activities. Second, the lack of standardized reports of physical activity and stress 
levels among studies did not allow a more quantitative analysis (i.e., meta-analysis). Third, half of the included studies examined the association between physical activity and stress in health workers. Although the crucial role of health professionals for the public health, other groups are still out of the focus of the research's lens such as farmers and school teachers. Fourth, no searches were performed in the gray literature, so some studies with the potential to be included may not be found.

In conclusion, some occupational groups are at higher risk of elevated stress levels regardless of physical activity practice in most studies. However, studies with robust methodologies and covering different groups of workers remain necessary.

\section{Conflict of interest}

The authors declare no conflict of interest.

\section{Author's contributions}

All authors participated in the conception and writing of the manuscript, analysis, data interpretation. Silva M, carried out the critical review of the content.

\section{References}

1. Lee IM, Shiroma EJ, Lobelo F, Puska P,Blair SN, Katzmarzyk PT. Effect of physical inactivity on major non-communicable diseases worldwide: an analysis of burden of disease and life expectancy. Lancet. 2012;380(9838):219-29.

2. World Health Organization. Global recommendations on Physical Activity for health. World Heal Organ; 2010. Available from: <https://apps.who.int/iris/rest/ bitstreams/52834/retrieve> [Novembro 2020].

3. Kyu HH, Bachman VF, Alexander LT, Mumford JE, Afshin A, Estep K, et al. Physical activity and risk of breast cancer, colon cancer, diabetes, ischemic heart disease, and ischemic stroke events: systematic review and dose-response metaanalysis for the Global Burden of Disease Study 2013. BMJ. 2016;354:i3857.

4. Holtermann A, Mortensen OS, Burr H, Søgaard K, Gyntelberg F, Suadicani P. The interplay between physical activity at work and during leisure time-risk of ischemic heart disease and all-cause mortality in middle-aged Caucasian men. Scand J Work Environ Health. 2009;35(6):466-74.

5. Skielboe AK, Marott JL, Dixen U, Friberg JB, Jensen GB. Occupational physical activity, but not leisure-time physical activity increases the risk of atrial fibrillation: The Copenhagen City Heart Study. Eur J Prev Cardiol. 2016;23(17):1883-93.

6. Coenen P, Huysmans MA, Holtermann A, Krause N, van Mechelen W, Straker LM, et al. Do highly physically active workers die early? A systematic review with metaanalysis of data from 193696 participants. Br J Sports Med. 2018;52(20):1320-6.

7. Hallman DM, Birk Jørgensen M, Holtermann A. On the health paradox of occupational and leisure-time physical activity using objective measurements: Effects on autonomic imbalance. PLoS One. 2017;12(5):e0177042.
8. Desouky D, Allam H. Occupational stress, anxiety and depression among Egyptian teachers. J Epidemiol Glob Health. 2017;7(3):191-8.

9. Song KW, Choi WS, Jee HJ, Yuh CS, Kim YK, Kim L, et al. Correlation of occupational stress with depression, anxiety, and sleep in Korean dentists: cross-sectional study. BMC Psychiatry. 2017;17(1):398.

10. Korshøj M, Lidegaard M, Kittel F, Van Herck K, De Backer $\mathrm{G}, \mathrm{De}$ Bacquer $\mathrm{D}$, et al. The relation of ambulatory heart rate with all-cause mortality among middle-aged men: a prospective cohort study. PLoS One. 2015;10(3):e0121729.

11. Holtermann A, Krause N, Van Der Beek AJ, Straker L. The physical activity paradox: six reasons why occupational physical activity (OPA) does not confer the cardiovascular health benefits that leisure time physical activity does. Br J Sports Med. 2018;52:149-150

12. Sorokin A V, Araujo CGS, Zweibel S, Thompson PD. Atrial fibrillation in endurance-trained athletes. Br J Sports Med. 2011;45(3):185-8.

13. Järvelin-Pasanen $S$, Sinikallio $S$, Tarvainen MP. Heart rate variability and occupational stress-systematic review. Ind Health. 2018;56(6):500-11.

14. Jackson EM. Stress relief: The role of exercise in stress management. ACSMs Health Fit J. 2013;17(3):14-9.

15. Moher D, Liberati A, Tetzlaff J, Altman DG. Preferred reporting items for systematic reviews and meta-analyses: The prisma statement. PLoS Med. 2009;6(7):e1000097.

16. Tooth L, Ware R, Bain C, Purdie DM, Dobson A. Quality of reporting of observational longitudinal research. Am J Epidemiol. 2005;161(3):280-8.

17. Von Elm E, Altman DG, Egger M, Pocock SJ, Gøtzsche PC, Vandenbroucke JP. The Strengthening the Reporting of Observational Studies in Epidemiology (STROBE) statement: guidelines for reporting observational studies. Ann Intern Med. 2007;147(8):573-7.

18. Van Uffelen JGZ, Wong J, Chau JY, Van der Ploeg HP, Riphagen I, Gilson ND, et al. Occupational sitting and health risks: a systematic review. Am J Prev Med.2010;39(4):379-88.

19. Driesman AS, Strauss EJ, Konda SR, Egol KA. Factors associated with orthopaedic resident burnout: a pilot study. J Am Acad Orthop Surg. 2020;28(21):900-6.

20. Larsson K, Ekblom O, Kallings V L, Ekblom M, Blom V. Job demand-control-support model as related to objectively measured physical activity and sedentary time in working women and men. Int $\mathrm{J}$ Environ Res Public Health. 2019;16(18):3370.

21. Marquez DX, Bustamante EE, Kozey-Keadle S, Kraemer J, Carrion I. Physical activity and psychosocial and mental health of older caregivers and non-caregivers. Geriatr Nurs. 2012;33(5):358-65.

22. Meina M, Ratajczak E, Sadowska M, Rykaczewski K, Dreszer J, Bałaj B, et al. Heart rate variability and accelerometry as classification tools for monitoring perceived stress levels-a pilot study on firefighters. Sensors (Basel). 2020;20(10):2834.

23. Schilling R, Colledge F, Puehse U, Gerber M. Stressbuffering effects of physical activity and cardiorespiratory fitness on metabolic syndrome: A prospective study in police officers. PLoS One. 2020;15(7):e0236526.

24. De Cocker K, Ketels M, Bennie JA, Clays E. Is device-based total and domain-specific sedentary behaviour associated with psychological distress in Flemish workers? Eur J Public Health. 2020;31(1):151-6. 
25. Tejada BM, Jastrow N, Poncet A, Le Scouezec I, Irion O, Kayser B. Perceived and measured physical activity and mental stress levels in obstetricians. Eur J Obstet Gynecol Reprod Biol. 2013;171(1):44-8.

26. Roskoden FC, Krueger J, Vogt LJ, Gärtner S, Hannich HJ, Steveling A, et al. Physical Activity, Energy Expenditure, Nutritional Habits, Quality of Sleep and Stress Levels in Shift-Working Health Care Personnel. PLoS One. 2017;12(1): e0169983.

27. Low JM, Tan MY, See KC, Aw MM. Sleep, activity and fatigue reported by Postgraduate Year 1 residents: a prospective cohort study comparing the effects of night float versus the traditional overnight on-call system. Singapore Med J. 2018;59(12):652-5.

28. Kitano N, Kai Y, Jindo T, Tsunoda K, Arao T. Compositional data analysis of 24-hour movement behaviors and mental health in workers. Prev Med Reports. 2020;20:101213.

29. Mendelsohn D, Despot I, Gooderham PA, Singhal A, Redekop GJ, Toyota BD. Impact of work hours and sleep on well-being and burnout for physicians-in-training: the Resident Activity Tracker Evaluation Study. Med Educ. 2019;53(3):306-15.
30. Myrtek M, Fichtler A, Strittmatter M, Brugner G. Stress and strain of blue and white collar workers during work and leisure time: results of psychophysiological and behavioral monitoring. Appl Ergon. 1999;30(4):341-51.

31. Gilson ND, Hall C, Holtermann A, Van der Beek AJ, Huysmans MA, Mathiassen SE, et al. Sedentary and Physical Activity Behavior in "Blue-Collar" Workers: A Systematic Review of Accelerometer Studies. J Phys Act Health. 2019;16(11):1060-9.

32. Holtermann A, Straker L, Lee I-M, van der Beek AJ, Stamatakis E. Long overdue remarriage for better physical activity advice for all: bringing together the public health and occupational health agendas. Br J Sports Med. 2020; 54(23):1377-8.

33. Brown WJ. Is remarriage of public health and occupational health advice on physical activity really necessary? Br J Sports Med. 2020; 54(23):1379-80.

Received: 02/07/2021

Approved: 08/11/2021

\section{Quote this article as:}

Marins MF, Silva BS, Feter N, Silva MC. Objectively-measured physical activity and stress levels in workers: a systematic review physical activity and stress in workers. Rev Bras Ativ Fís Saúde. 2021;26:e0232. DOI: 10.12820/rbafs.26e0232 